\title{
The Most Massive Heartbeat: Finding the Pulse of $\iota$ Orionis
}

\author{
Herbert Pablo ${ }^{1}$, Noel Richardson ${ }^{2}$, Jim Fuller ${ }^{3,4}$, \\ Anthony F. J. Moffat ${ }^{1}$, BEST and Ritter Observing team \\ ${ }^{1}$ Département de physique and Centre de Recherche en Astrophysique du Québec (CRAQ), \\ Université de Montréal, C.P. 6128, \\ Succ. Centre-Ville, Montréal, Québec, H3C 3J7, Canada \\ email:hpablo@astro.umontreal.ca \\ ${ }^{2}$ Ritter Observatory, Department of Physics and Astronomy, The University of Toledo, Toledo, \\ OH 43606-3390, USA \\ ${ }^{3}$ TAPIR, Walter Burke Institute for Theoretical Physics, Mailcode 350-17, Caltech, Pasadena, \\ CA 91125, USA \\ ${ }^{4}$ Kavli Institute for Theoretical Physics, Kohn Hall, University of California, Santa Barbara, \\ CA 93106, USA
}

\begin{abstract}
Orionis is a massive binary system consisting of O9III + B1 III/IV stars. Though the system has been well studied, much about its fundamental properties have been difficult to determine. In this paper we report on the discovery of the heartbeat phenomenon in $\iota$ Orionis making it the most massive heartbeat system currently known. Using this phenomenon we have found empirical values for the masses and radii of both components. Moreover, we report the detection of tidally induced oscillations in an O-type star for the first time. These discoveries open a new avenue for exploring asteroseismology in massive stars.
\end{abstract}

Keywords. (stars:) binaries (including multiple): close, stars: fundamental parameters (classification, masses, radii), stars: oscillations (including pulsations), stars: individual: $\iota$ Ori

\section{Introduction}

Understanding stellar evolution is essential as it is stars which are the building blocks on which galaxies, and by extension, the universe as a whole is built. It is even more important that we understand these processes in massive stars as these stars will explode and pollute the interstellar medium with metals. Unfortunetely, massive stars have comparatively short lifetimes compared to lower mass stars, making ensemble statistical studies extremely difficult. Another avenue of exploration is through the use of asteroseismology. By studying the oscillations produced within stars, we can interpret their interior structure, and from that their evolutionary state. However, this channel has also been largely restricted as there are only handful of $\mathrm{O}$ stars which are known to pulsate (see Buysschaert et al. 2015; Pablo et al. 2015, and references therein).

Further complicating matters is that almost all massive stars are born as binary stars (Sana et al. 2012, Sana et al. 2014, Aldoretta et al. 2015) and it has been shown that only $24 \%$ will not interact or merge during their lives. This makes it imperative that we include binary systems when trying to understand evolution in massive stars. Studying binary systems, though, is not a simple proposition. While it is true that in such systems we are able to determine fundamental parameters, this typically requires close eclipsing binary systems. Such constraints have led to only $\approx 50 \mathrm{O}$ star binaries for which fundamental parameters are known (Gies et al. 2012). These constraints are also problematic as many 
such binaries will have had interactions making it difficult to determine the evolutionary history of the system.

Virtually all these problems could be mitigated, however, by the discovery of a new class of binary systems. This class first discovered with the Kepler space telescope (Thompson et al. 2012), are commonly labeled heartbeat systems, as their light curves' bear qualitative similarities to the "normal sinus rhythm" signal of an electrocardiogram. This peculiar heartbeat occurs in eccentric binary systems, which show strong evidence of ellipsoidal variation only at periastron, when the stars are closest. Due to the strong dependence of this heartbeat phenomenon on inclination it is possible to use such systems to derive fundamental parameters, even in the absence of eclipses. A further consequence of the stars' close proximity at periastron, is the ability to induce tidally excited oscillations (TEOs) in their companion. This affords us the opportunity to do asteroseismology within binary systems.

The only drawback is the lack of known systems. Due to the high binary fraction of $\mathrm{O}$ stars and their short lifetimes (and consequently higher eccentricities), this would seem to be due largely to selection bias. Because this phenomenon is often only a few parts per thousand, the heartbeat phenomenon is very difficult to observe from the ground in addition to requiring long time-baseline photometry. The only space missions which have heretofore observed this effect, CoRoT and Kepler, have looked at a total of $6 \mathrm{O}$ stars (though this will undoubtedly change with K2). However in 2013, a new network of nanosatellites, called BRIght Target Explorer (BRITE)-Constellation, was launched to address this sort of problem. Its mission is to look at the brightest stars in the sky with high-precision, long time-baseline observations Weiss et. al, (2014). As these stars are often the most intrinsically luminous stars it is an excellent resource for studying massive star variability, such as the heartbeat phenomenon.

In these proceedings we will give an overview of the results of Pablo et al. (2017, accepted) who have identified $\iota$ Ori a massive O9 III + B1 III/IV eccentric binary $(e=$ 0.76) with $P_{\text {orb }}=29.13376 \mathrm{~d}$ as the most massive heartbeat star ever weighed. Using light curves from two separate BRITE observing runs, in addition to radial velocities from Marchenko et al. (2000) as well as new observations from the $1 \mathrm{~m}$ Ritter observatory telescope (University of Toledo) we were able to obtain a full binary solution. In addition, we were able to identify 5 separate TEOs, the first ever observed in an O star. Finally we will discuss the implications of this work as it relates massive star evolution.

\section{Overview}

Binary Simulation and fitting.

The prevalence of TEOs in heartbeat systems is extremely useful for asteroseismic analysis, but has a single important drawback when attempting to find a binary solution: the oscillations are not canceled out when the light curve is phased on the orbital period as they occur at multiples of the orbital frequency. This is only an issue at periastron, but it is a significant one as the frequencies (given in detail in Pablo et al. (2017)) are as large as $10 \%$ of the heartbeat effect. Therefore, the TEOs which had the largest influence on the phased light curve were removed using a least squares fit on the phased light curve, where the heartbeat itself is masked. This is explained in greater detail in Pablo et al. (2017). Once the oscillations were removed the system was run through the binary simulation code PHOEBE (Prsa \& Zwitter 2005). First, the system was fit using a Levenburg-Marquardt algorithm to determine a set of realistic parameters which resulted in an adequate fit to both the light and radial velocity curves. These values were used to initialize our Monte Carlo Markov Chains (MCMC). We then used these chains to probe 

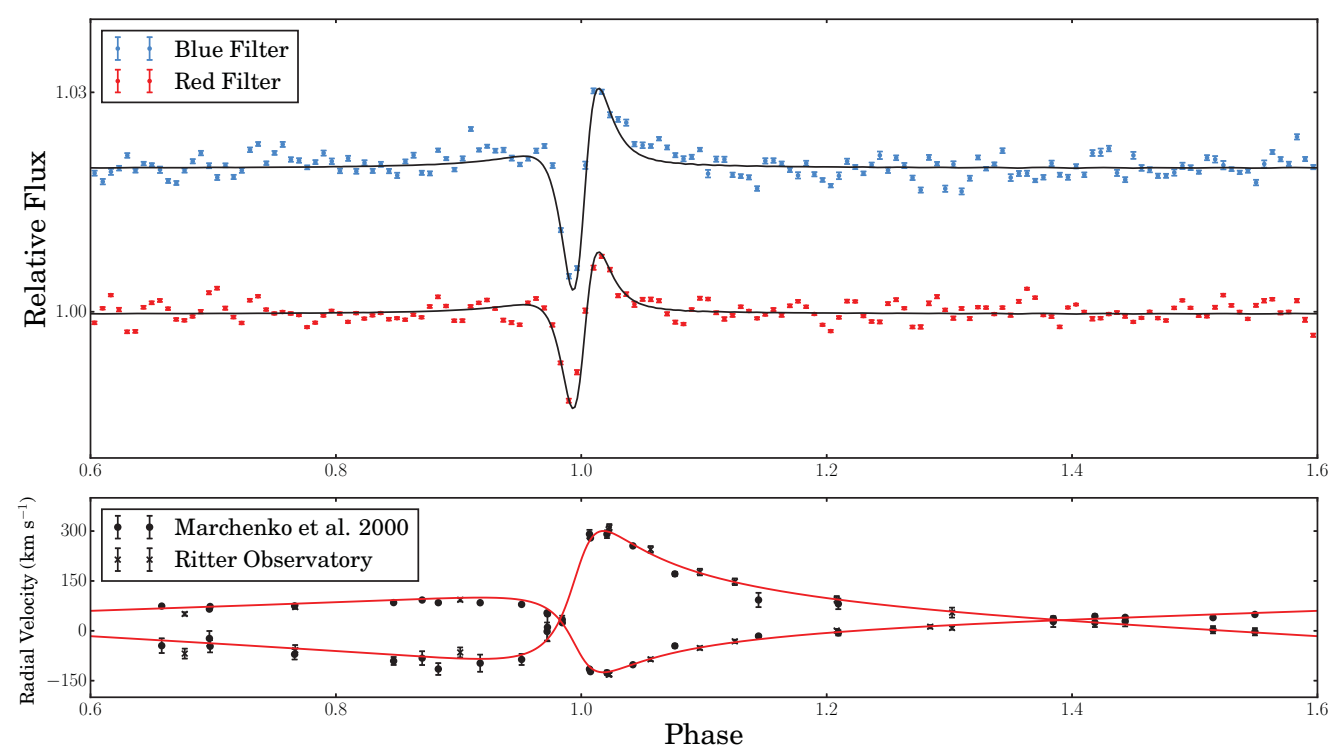

Figure 1. Simulation of 50 percentile values (black) derived from the simultaneous MCMC fit of phase-binned BRITE light curves (top) and radial velocities (bottom). The frequencies have not been removed, to demonstrate the presence of TEOs.

the parameter space, identifying degeneracies as well as determining the extent of the global minimum for error calculation. Our MCMC implementation is achieved through the Python package EMCEE (Foreman-Mackey et al. 2013). Because this procedure is computationally expensive several parameters were not fit, namely period and time of periastron as no noticeable change could be seen in the new data from the values given in Marchenko et al. (2000). The fit is given in Fig. 1.

This equates to a primary star with parameters $M_{\mathrm{p}}=23.18_{-0.53}^{+0.57} M_{\odot}, R_{\mathrm{p}}=9.10_{-0.10}^{+0.12} R_{\odot}$ and a secondary with parameters $M_{\mathrm{s}}=13.44_{-0.30}^{+0.30} M_{\odot}, R_{\mathrm{s}}=4.94_{-0.23}^{+0.16} R_{\odot}$. When compared with the expected values given for O stars (Martins 2005) and B stars (Nieva \& Pryzbilla 2014) the only noticeable difference is in the luminosity class. The primary values put it likely as a luminosity class IV as its parameter values fall between that of class III and V. For the secondary, while it is possible that it has luminosity class of $\mathrm{IV}$, there is no discernible difference in its parameters from that of a class V star of the same spectral type. The only other unusual quantity is the temperature of the secondary, $T_{\text {eff }}=18319_{-758}^{+531} \mathrm{~K}$. This value is almost $10000 \mathrm{~K}$ lower than would be expected from a B1 star. While it is clear from the MCMC calculations that this value is more favorable and is independent of range or initial value, it is also clear that this temperature is highly unlikely. The most likely explanation, therefore is that the two BRITE filters are not enough to get an accurate value of the temperature in the absence of eclipses. Instead, there is likely a degeneracy with another parameter which was not fit. See Table 2 in Pablo et al. (2017) for a full list of binary parameters.

Fourier Analysis \& Tidally Excited Oscillations.

Due to a 6 month gap between two separate campaigns on the Orion field, the frequency analysis was done on each campaign separately as well as on data from each filter. This resulted in 4 separate datasets from which frequencies were determined. In total 5 frequencies were found to be multiples of the orbital frequency $f_{\text {orb }}=0.0343244(2)$ $\mathrm{d}^{-1}$. The frequencies, shown in Fig. 2 , are at $6,23,25,27,33 f_{\text {orb }}$. As can be seen these frequencies appear in more than one color and campaign. The complete list of frequencies 


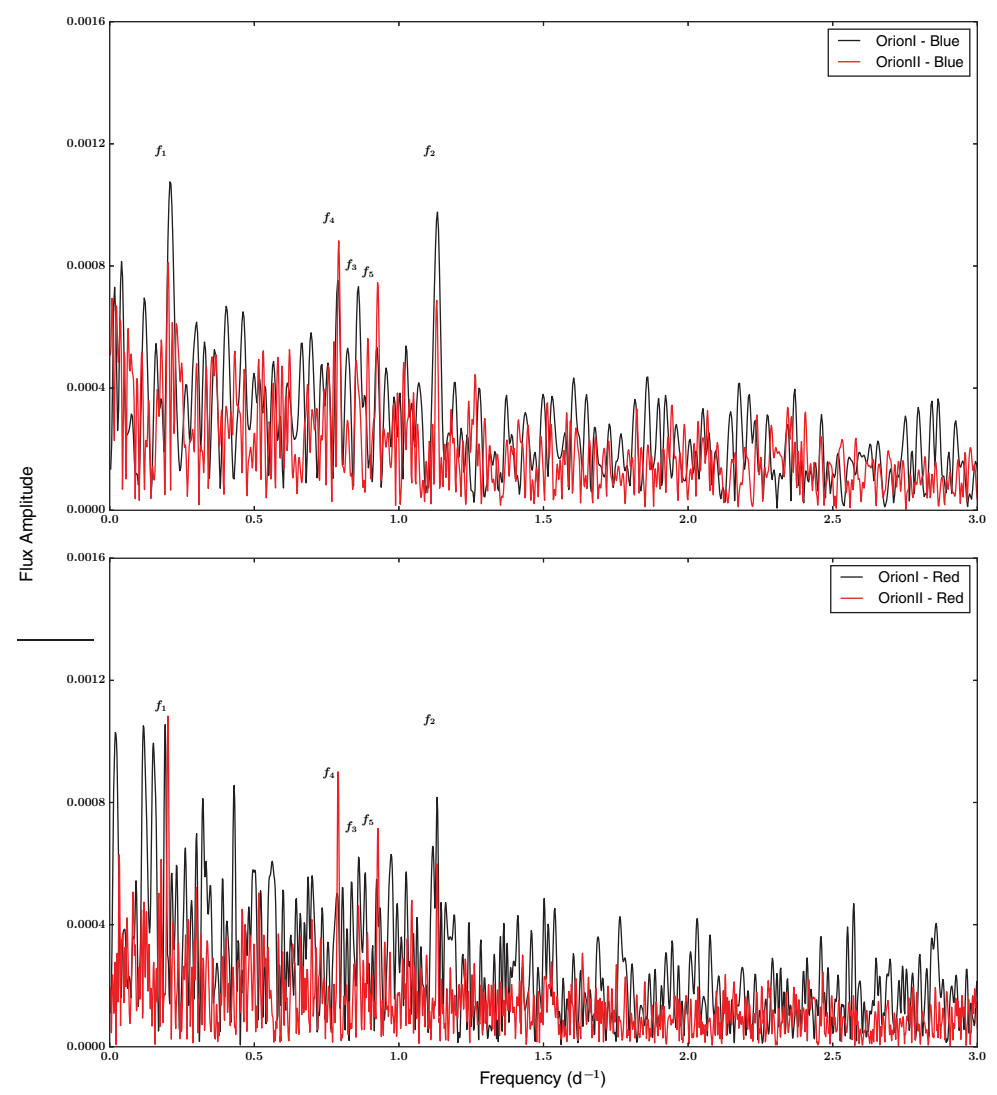

Figure 2. Discrete Fourier spectrum, from the blue (top) and red (bottom) filter of the OrionI and OrionII BRITE observing campaigns. The tidally excited oscillations are marked.

and their parameters (see Pablo et al. (2017), Tab. 3) also suggest that the amplitudes and phases are largely stable between observation epochs.

From the binary parameters, stellar models were calculated through the us of the MESA stellar evolution code (Paxton et al., 2011, Paxton et al., 2013, Paxton et al., 2015). Based on these models oscillation modes were found using the non-adiabatic version of the GYRE pulsation code (Townsend \& Teitler 2013). The oscillations produced were able to adequately reproduce 4 of the 5 frequencies as tidally induced $l=2$ modes. The one frequency which does not fit the models is the 6th harmonic, which is at a significantly lower frequency than all others. It is possible that this frequency just happens to be close to an orbital harmonic but is not tidally induced. However, its persistence across datasets makes this idea unlikely. A possible explanation is that the pulsation arises from non-linear interactions between TEOs, a common occurrence in heartbeat stars (Fuller et al., 2012, Hambleton et al., 2013, O'Leary et al., 2014, Guo et al. 2016). These models have also led to the estimation of a tidal dissipation rate, $Q_{\text {tide }}=4 \times 10^{4}$, for the primary star. While this result is model dependent, it represents the first empirical estimate of the tidal dissipation rate in an $\mathrm{O}$ star.

\section{Implications}

The determination of fundamental parameters for $\iota$ Ori is significant, if only because there are very few $\mathrm{O}$ type systems for which these parameters are known. What makes 
the system even more interesting, though, is that these parameters are known in a system with clear pulsations. This gives us the ability to constrain and test asteroseismic properties, which has been underutilized in massive stars simply due to the dearth of systems. While this analysis is still in its infancy $\iota$ Ori has already allowed for unprecedented asteroseismic calculations in massive stars, and these results will only improve as more data are obtained.

Beyond the results of $\iota$ Ori are the avenues this opens up for the study of massive star evolution. Heartbeat stars provide us the opportunity to study the interior structure of massive stars in a manner which, up to now, has never been done. What's more these systems are not rare. While $\iota$ Ori is the only system which has currently been published several more are known in the BRITE sample including $\epsilon$ Lupi, the only massive binary where both components are magnetic (Pablo et al. in preparation). Moreover, it is likely that several systems exist in the literature which have been overlooked as heartbeat systems were only discovered in 2011. Over the coming years analysis of these heartbeat systems should redefine our knowledge of how massive stars evolve, as they not only give us access to asteroseismology, but provide fundamental parameters for non-interacting massive stars from which to constrain many other properties such as mass loss rates and magnetism.

\section{References}

Aldoretta, E. J., Caballero-Nieves, S. M., Gies, D. R., Nelan, E. P., \& Wallace, D. J., 2015, AJ, 149,26

Buysschaert, B., Aerts, C., Bloemen, S., Debosscher, J., \& Neiner, C., 2015, MNRAS, 453, 89

Foreman-Mackey, D., Hogg, D. W., Lang, D., \& Goodman, J., 2013, PASP, 125, 306

Fuller, J., \& Lai, D., 2012, MNRAS, 420, 3126

1995, Meteoritics, 30, 490

Guo, Z., Gies, D. R., \& Fuller, J. 2016, ApJ, 834, 59

Hambleton, K. M., Kurtz, D. W., Prša, A., Guzik, J. A., Pavlovski, K., et al., 2013, MNRAS, 434,925

Marchenko, S. V., Rauw, G., Antokhina, E. A., Antokhin, I. I., Ballereau, D. et al. 2000, MNRAS, 317,333

O'Leary, R. M. \& Burkart, J. 2014, MNRAS, 440, 3036

Pablo, H., Richardson, N. D., Moffat, A. F. J., Corcoran, M., Shenar, T. et al. 2015, ApJ, 809, 134

Pablo, H., Richardson, N. D., Fuller J., Moffat, A. F. J., Rowe, J. et al. 2017, MNRAS, accepted

Paxton, B., Bildsten, L., Dotter, A., Herwig, F., Lesaffre, P. et al. 2011, ApJs, 192, 3

Paxton, B., Cantiello, M., Arras, P., Bildsten, L., Brown, E. F. et al. 2013, ApJs, 208, 4

Paxton, B., Marchant, P., Schwab, J., Bauer, E. B., Bildsten, L. et al. 2015, ApJs, 220, 15

Sana, H., de Mink, S. E., de Koter, A., Langer, N. Evans, C. J. et al. Science, 337, 444

Sana, H., Le Bouquin, J.-B., Lacour, S., Berger, J.-P., Duvert, G. et al. 2014, ApJs, 215, 15

Thompson, S. E., Everett, M., Mullally, F., Barclay, T., Howell, S. B. et al. 2012, ApJ, 2012, 753

Townsend, R. H. D. \& Teitler, S. A. 2013, MNRAS, 435, 3406

Weiss, W. W., Rucinski, S. M., Moffat, A. F. J., Schwarzenberg-Czerny, A., Koudelka, O. F. et al. 2014, PASP, 126, 573 